Article

\title{
Transmission Power Adaption for Full-Duplex Relay-Aided Device-to-Device Communication
}

\author{
Hui Dun, Fang Ye* and Yibing Li \\ College of Information and Communication Engineering, Harbin Engineering University, Harbin 150001, \\ Heilongjiang Province, China; m18846198960@163.com (H.D.); yibing0920@126.com (Y.L.) \\ * Correspondence: yefang0923@126.com; Tel.: +86-133-0460-5678
}

Academic Editor: Angel Garrido

Received: 1 December 2016; Accepted: 3 March 2017; Published: 9 March 2017

\begin{abstract}
Device-to-device (D2D) communications bring significant improvements of spectral efficiency by underlaying cellular networks. However, they also lead to a more deteriorative interference environment for cellular users, especially the users in severely deep fading or shadowing. In this paper, we investigate a relay-based communication scheme in cellular systems, where the D2D communications are exploited to aid the cellular downlink transmissions by acting as relay nodes with underlaying cellular networks. We modeled two-antenna infrastructure relays employed for D2D relay. The D2D transmitter is able to transmit and receive signals simultaneously over the same frequency band. Then we proposed an efficient power allocation algorithm for the base station (BS) and D2D relay to reduce the loopback interference which is inherent due to the two-antenna infrastructure in full-duplex (FD) mode. We derived the optimal power allocation problem in closed form under the independent power constraint. Simulation results show that the algorithm reduces the power consumption of D2D relay to the greatest extent and also guarantees cellular users' minimum transmit rate. Moreover, it also outperforms the existing half-duplex (HD) relay mode in terms of achievable rate of D2D.
\end{abstract}

Keywords: device-to-device (D2D); relay aided; full-duplex (FD); power allocation; independent power constraint

\section{Introduction}

Device-to-device (D2D) communication enables users in proximity to exchange information directly, without traversing to the base station (BS) or core network in two hops and has attracted increasing attention from both industrial and academic communities [1-7]. Due to the potential proximity gain, reuse gain, and hop gain, D2D communications can significantly increase system spectral efficiency and energy efficiency, which has been considered a promising candidate technique for next generation cellular networks [8].

Accordingly, many researches have focused on a D2D communications scheme that underlayings the cellular network infrastructure to increase the cellular capacity, improving the users' throughput, and extending the battery lifetime of user equipment (UE) [9-11]. However, the existing D2D communication scheme, such as the underlaying cellular spectrum, bring more complex interference environment to cellular users which have higher priority than the D2D users, and lead to deteriorative performance on quality of service for cellular users [12-14]. In particular, for cellular users in the cell edge who are susceptible to suffered deep fading or shadowing, it would be unadvisable to share an allocated spectrum with D2D peers [15-17].

To avoid the problem mentioned above, we outline a relay-aided D2D communication scheme to fulfill the transmission gap that affects cellular users which are in the cell edge or strongly-shadowed urban environment. Unlike existing works, a D2D transmitter in our scheme is ordered to aid a cellular 
downlink transmission by acting as a relay node between the BS and the cellular UE. In return, the D2D transmitter is allowed to directly communicate with the intended D2D receiver by underlaying the cellular downlink spectral resource [18-20].

Few works have been carried out so far to explore relaying-based D2D communication schemes. By scanning existing research papers, we can classify this relay-aided D2D communication schemes into two categories in terms of relay mode, namely, half-duplex (HD) and full-duplex (FD). In fact, few works have been carried out so far to explore HD relaying based scheme to transmit forward signals from BS to cellular users in two transmission phases. In this scheme, respective reception and transmission at the relay are allocated to one frame which is divided into double orthogonal time slots which are equivalent to a time division access way for the D2D relay node [21-26]. Then the superimposed signal, which is a linear weighted combination of cellular user's signal and the D2D signal is generated by the relay. The weight factor represents the power allocation for the cellular user which is a fraction of the total transmitting power of D2D relay. Therefore, the residual power is used for broadcasting the D2D signal in the second time slot.

The drawback of HD is lower efficiency in spectrum and energy. Therefore, as a replacer for HD relay, the FD mode is more applicable in practice to transmit and receive signals simultaneously on the same frequency resource. For the sake of communication bi-directional for relay, a two-antenna infrastructure is typically deployed to facilitate spectral efficiency in practice. However, FD relay is more susceptible to loopback interference which is generated from transmitting antenna to receiving antenna of relay nodes. Although few works have been done to eliminate the inter-relay interference, it cannot be ultimately mitigated because even the residual loopback interference is significant when compared to the white Gaussian noise [27-29]. For this reason, the power split factor should be adapted cautiously in FD mode. Moreover, it must be made clear that due to the FD mode being enable relay to forward two components signals simultaneously on the same spectral band, the cellular user's signal and D2D signal will treat each other as interference.

Many works have been carried out to explore relaying-based D2D communication schemes. In [21], the authors outline an HD relaying-based D2D scheme by operating on the same frequency band as cellular user in frequency domain by splitting a frame into double transmitting phases in the time domain. In this scheme, because the BS transmit signal to the cellular user takes place in one half of a frame, the achievable transmission rate is dimidiated in this mode. Then to compensate for the cellular users' capacity loss, the D2D relay has to allocate almost all of its transmitting power to forward cellular signals when the direct link between the BS and cellular users is good. Then this scheme will degenerate to a conventional relaying mode. In contrast, when the direct link is in deep fading or shadowing, it also cannot give a significantly increasing transmission rate. Furthermore, the authors describe explicitly that the BS and the D2D relay should use maximum transmitting power as they can maintain the transmit capacity of the cellular user, which leads to low energy efficiency for the system.

Alternatively, the FD mode as explicitly described in [27-29] relays cellular signals and the D2D component to the respective receiver simultaneously. Unfortunately, the self-interference cannot be eliminated perfectly and, consequently, the potential existence of residual interference is not negligible. The work that has been done in [27] advocates a new FD mode, in which the aggregate power of the system is defined as a constant. That makes the D2D relay much more able to use transmitting power than one when the power is normalized. By this means, they can get a significant capacity increase and a closed form of expression of the optimal power at BS and D2D relay by adding a power equation. However, it is more realistic to consider the BS and D2D relay with their own transmission power constraint.

In contrast to the aforementioned works, the contributions of this paper are summarized as follows:

(1) The main contribution of the article is to draft a novel D2D relaying-aided scheme to improve the transmitting rate of a cellular user who is in deep fading or shadowing. Unlike the works that 
have been done before, our scheme guarantees cellular user' quality of service in terms of the minimum rate requirement. This means that the performance of the cellular user is improved rather than deteriorated or just maintained, as done in $[13,21]$.

(2) We consider an independent power constraint for the BS and D2D relay respectively rather than under the aggregate power constraint as in [27]. Although we use two inequalities to replace one equation, we also derived a closed form expression of the optimal allocated power and the power split factor.

(3) By making comparisons with traditional direct communication of cellular mode and HD relaying mode, the FD mode we proposed could use the minimum transmitting power to make sure the cellular' rate requirement with also achieve the D2D rate as far as possible. It also has been demonstrated that the approach we proposed is more prominent in terms of spectral efficiency and power efficiency, in the situation that the residual loopback interference generating the D2D relay is controlled at a tolerable level.

The rest of the paper is organized as follows: we describe the system model and scheme of the proposed FD relaying assisted D2D communication by underlaying the downlink frequency resource of a cellular network in Section 2. The optimal power adaption strategies for guaranteeing the rate requirement of cellular user versus benchmark HD mode are evaluated in Section 3. Simulations have been obtained are presented in Section 4. Finally, the conclusions are drawn in Section 5.

\section{System Model}

In Figure 1 we consider a two-antenna deployment scenario, where a macro BS is intended to communicate with a cellular user who is in the edge of traditional cell and suffering severe deep fading or shadowing. This means the direct link between macro BS and cellular is weak and unable to guarantee the rate requirement of cellular user. Moreover, there is a pair of D2D users located nearby the cellular user and there is a good channel quality from BS and cellular respectively. We assume that the transmitter of the D2D pair is equipped with isolated receiving and transmitting antennas and caches the date that intends to send to the D2D receiver. We can automatically see that the system performance will be beneficial from deploying D2D transmitter as a relay for BS and the cellular user. We assume that the D2D communication is allowed to share the downlink spectral resource allocated to the cellular user while remaining under the control of the BS. We set the D2D relay to operate in FD mode, suffering from the backhaul interference is inevitable due to the simultaneously receiving and transmitting signals which is an inherent nature of FD mode. It also should be made clear that this scenario corresponds to the situation that radio resource management has taken place and spectrum resources have been allocated for a particular BS to serve a particular cellular user.

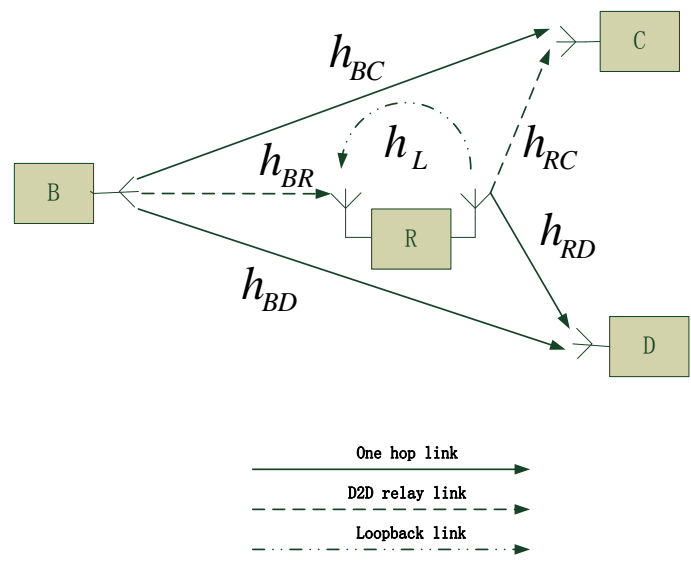

Figure 1. Relay-aided device-to-device (D2D) communication model in full-duplex (FD) mode. 
We assume that the system operates in the downlink in frequency division duplex (FDD) mode with a bandwidth of $W \mathrm{~Hz}$. We denote the channel impulse response for the link from BS to cellular user, BS to the relay, namely, the D2D transmitter, from BS to the D2D receiver, from the relay to cellular, from the relay to the D2D receiver and the loopback link from the transmitting antenna to the receiving antenna as $h_{B C}, h_{B R}, h_{B D}, h_{R C}, h_{R D}$ and $h_{L}$ respectively. These are all complex variables. The system is parametrized with channel gain-to-noise ratios, which are defined as:

$$
\begin{gathered}
\gamma_{B R}=\left|h_{B R}\right|^{2} / \sigma^{2}, \gamma_{B C}=\left|h_{B C}\right|^{2} / \sigma^{2}, \gamma_{B D}=\left|h_{B D}\right|^{2} / \sigma^{2} \\
\gamma_{R C}=\left|h_{R C}\right|^{2} / \sigma^{2}, \gamma_{R D}=\left|h_{R D}\right|^{2} / \sigma^{2}, \gamma_{L}=\left|h_{L}\right|^{2} / \sigma^{2}
\end{gathered}
$$

Where $\sigma^{2}$ denotes the variance of the zero-mean additive white Gaussian noise (AWGN). As we can see, each of these variables actually corresponds to the signal-to-noise ratio (SNR) of one-hop transmission over the particular channel with maximum transmission power. In other words, we set $p_{B}=1$ and $p_{R}=1$. All of the channels we used are modeled to be narrow band quasi-static state and frequency flat fading. Note that we assume that the nodes in our scenario are generally stationary or moving at moderate speed to allow channel state information acquisition.

The transmitting power of BS and relay node are denoted by $p_{B}$ and $p_{R}$ respectively. These have been normalized and are subject to independent constraints by $0 \leq p_{B} \leq 1$ and $0 \leq p_{R} \leq 1$ instead of aggregate transmitting power constraint $p_{B}+p_{R}=2$ as does in [27]. We assume that the D2D relay adapts amplify-and-forward protocol to aid the downlink transmission of a cellular user and the relay uses a fraction of its transmitting power to forward the cellular user's signals.

\section{FD Relay-Aided D2D Communication Scheme}

Before making an explicit description of the proposed relaying based D2D scheme, we review the traditional cellular direct transmission (DT) mode. In a traditional cellular network, the cellular users have no choice but to communicate with the BS even the DT link suffers from severely deep fading or shadowing. Unfortunately, this situation does not happen in a small probability. When the cellular users are located in the cell edge or urban environment, it will be a frequent occurrence. Therefore, DT links from the BS to cellular users in a conventional cellular mode will lead to a poor performance of the system in this situation, and might even cause high outage probability or call drop for cellular users. Assume frequency-division multiple-access technology is used for the cellular downlink transmissions in DT mode. At any time instant, the BS transmits symbols to the cellular user over the allocated frequency band. Therefore, the achievable rate of direct link is given by:

$$
R_{B C}^{D T}=W \log _{2}\left(1+p_{B} \gamma_{B C}\right)
$$

where $W$ corresponds to the width of the subchannel. Let $R^{r e}$ represent the rate requirement of the cellular user. When the channel impulse response $h_{B C}$ suffers from deep fading or shadowing, it will lead to the situation that the achievable rate of the DT link will fall below the rate requirement even the BS use maximum transmitting power with $p_{B}=1$, namely:

$$
R^{r e}>W \log _{2}\left(1+\gamma_{B C}\right)
$$

Hence, for the sake of fulfilling the requirement rate of cellular user, we point out that the desired channel to noise ratio $\gamma$ should satisfy:

$$
\gamma \geq \frac{\left(2^{R^{q e} / W}-1\right)}{p_{B}}
$$

From the analysis above, we conclude that the conventional DT mode can do nothing to satisfy the cellular user's quality of service requirement in terms of transmitting rate when the DT link is 
weak. Therefore, we aim to find a novelty D2D relaying-aided scheme to guarantee the cellular user's rate requirement.

In order to improve spectral efficiency, throughput and energy efficiency, BS allows UE who are in proximity to communicate directly without the help of BS. Moreover, for the sake of improving the poor performance of conventional cellular mode in which the cellular users suffer severely from deep fading or shadowing, we outline an FD relaying based D2D communication scheme to allow the downlink transmission from BS to cellular user via D2D relay and D2D direct communication from D2D relay to D2D receiver take place at the same instant. In our system model, it is assumed that the D2D relay is equipped with two isolate antennas.

We now describe the relaying based D2D protocol. Let us denote $x(i)$ and $d(i)$ as the signals transmitted by BS and D2D relay, namely, D2D transmitter at time instant $i$. Then we have:

$$
\begin{gathered}
E\left\{|x(i)|^{2}\right\}=p_{B} \\
E\left\{|d(i)|^{2}\right\}=(1-\alpha) p_{R}
\end{gathered}
$$

in which $E\{\cdot\}$ denotes the expectation of variable and $\alpha$ represents the power split factor at relay. We assume that the D2D relay is willing to share $\alpha(0 \leq \alpha \leq 1)$ fraction of its power to assist the cellular user's downlink transmission. Then the residual $(1-\alpha)$ fraction is used for its own signal transmission. In addition, the system will degrade to a conventional relay forward mode or a D2D underlaying mode when the power split factor $\alpha$ is equal to zero or one respectively. Furthermore, considering the loopback interference from the relay transmitting antenna to the receiving antenna, the signal $r(i)$ received at the D2D relay and the transmitting signal $t(i)$ by D2D relay can be formulated as:

$$
\begin{aligned}
& r(i)=h_{B R} x(i)+h_{L} t(i)+n(i) \\
& t(i)=g r(i)+\sqrt{(1-\alpha) p_{R}} d(i)
\end{aligned}
$$

where $n(i)$ is the AWGN at relay with mean value $E\left\{|n(i)|^{2}\right\}=\sigma^{2}$. From this, we know that the power of receiving signal at $\mathrm{D} 2 \mathrm{D}$ relay is:

$$
E\left\{|r(i)|^{2}\right\}=p_{B}\left|h_{B R}\right|^{2}+p_{R}\left|h_{L}\right|^{2}+\sigma^{2}
$$

$g$ represents the normalized amplify factor by the D2D relay with adopting amplify forward protocol which means that the relay will amplify the input signal by factor $g$. It can be given by:

$$
g=\sqrt{\frac{\alpha p_{R}}{p_{B}\left|h_{B R}\right|^{2}+p_{R}\left|h_{L}\right|^{2}+\sigma^{2}}}
$$

in which $\alpha p_{R}$ is denoted as the power used for forwarding cellular user' signals. Naturally, we also have the aggregate power consumption at D2D relay as $E\left\{|t(i)|^{2}\right\}=p_{R}$. Thus, the signal-to-interference and noise ratios (SINR) $\gamma_{R}$ of the first hop at the relay can be formulated as:

$$
\gamma_{R}=\frac{p_{B}\left|h_{B R}\right|^{2}}{p_{R}\left|h_{L}\right|^{2}+\sigma^{2}}
$$

Then we let the numerator and denominator be divided by $\sigma^{2}$, the SINR can be rewritten as:

$$
\gamma_{R}=\frac{p_{B} \gamma_{B R}}{p_{R} \gamma_{L}+1}
$$


From the Equation (8), we can see that the transmitting signals of D2D relay are composed by two portions. The first portion contains the signal $x(i)$ transmitting for cellular user and the second portion is the signal component sending to the D2D receiver using the remaining $(1-\alpha)$ fraction power. When decoding respective signals at the receivers, the cellular user and D2D receiver will treat the signal component designated for the other as interference. We get the receiver signal at cellular user and D2D receiver as follows:

$$
\begin{aligned}
& y_{C}(i)=h_{B C} x(i)+h_{R C} t(i)+n(i) \\
& y_{D}(i)=h_{B D} x(i)+h_{R D} t(i)+n(i)
\end{aligned}
$$

In FD mode, the cellular user decodes the relayed signal $t(i)$ from $y_{C}(i)$ and the weak DT link signal $x(i)$ is treated as interference. Therefore, as above, the SINR of the second hop at the cellular user $\gamma_{C}(i)$ can be formulated as:

$$
\gamma_{C}=\frac{\alpha p_{R} \gamma_{R C}}{p_{B} \gamma_{B C}+(1-a) p_{R} \gamma_{R C}+1}
$$

The instantaneous end-to-end SINR of one symbol transmitting from BS to cellular user in FD mode can be expressed as [28]:

$$
\gamma_{F D}=\frac{\gamma_{R} \gamma_{D}}{\gamma_{R}+\gamma_{D}+1}
$$

In our model, these expressions we deduce are explicitly including the effects of the self-interference and the overheard direct link. Then to guarantee the rate requirement of the cellular user with the proposed spectrum sharing protocol, the SINR of the system should be greater or equal to the requirement of SINR in the conventional DT mode as follows:

$$
\gamma_{F D} \geq \gamma
$$

As before, the SINR of D2D receiver can also be formulated as:

$$
\gamma_{D}=\frac{(1-\alpha) p_{R} \gamma_{R D}}{p_{B} \gamma_{B D}+\alpha p_{R} \gamma_{R D}+1}
$$

Finally, we can get the achievable rates for the cellular and D2D links respectively, as:

$$
\begin{gathered}
R_{C}^{F D}=W \log _{2}\left(1+\gamma_{F D}\right) \\
R_{D}^{F D}=W \log _{2}\left(1+\gamma_{D}\right)
\end{gathered}
$$

\section{The Optimal Power Adaption Strategies}

Let us then define the objective function as:

$$
\begin{gathered}
\left(p_{B}^{*}, p_{R}^{*}, \alpha^{*}\right)=\arg \max _{\left(p_{B}, p_{R}, \alpha\right)} R_{D}^{F D} \\
\text { s.t. } \quad R_{C}^{F D}=R^{r e} \\
0 \leq p_{B} \leq 1 \\
0 \leq p_{R} \leq 1 \\
0 \leq \alpha \leq 1
\end{gathered}
$$

Where $\left(p_{B}^{*}, p_{R^{\prime}}^{*} \alpha^{*}\right)$ represents the optimal power allocation for BS and the D2D relay and the power split factor for the FD relaying D2D. 
Next we propose transmission power adaptation as a technique to mitigate the effect of self-interference on D2D relay. Without optimal transmit power adaptation at the D2D relay, it implies that the D2D transmitter simply uses the maximum allowed power, i.e., $p_{R}=1$.

Remark 1: For extension of cellular coverage, the BS should always use the maximum transmission power, i.e., $p_{B}=1$. In this way, it will enlarge the SINR for the first relaying hop, namely $\gamma_{R}=p_{B} \gamma_{B R} /\left(p_{R} \gamma_{L}+1\right)$. Moreover, because typically we have $\gamma_{L} \gg \gamma_{B C}$, the worsening effect on $\gamma_{C}=\alpha p_{R} \gamma_{R C} /\left(p_{B} \gamma_{B C}+(1-\alpha) p_{R} \gamma_{R C}+1\right)$ will be insignificant with the maximum transmission power usage for BS. Thus, the best performance under constraint $0 \leq p_{B} \leq 1$ is achieved when $p_{B}=1$ and we may concentrate on power adaptation for D2D relay individually. Transmission power adaptation in FD relays is motivated by the observation that the end-to-end performance is limited by the weakest hop. Thus, if the limiting factor is the first hop due to excessive loop interference, the end-to-end performance can be, in fact, improved by decreasing the relay transmit power.

To solve the optimal problem, we first obtain $\gamma_{F D}=\gamma$ from $R_{C}^{F D}=R^{r e}$.

Remark 2: from (16), we know that $\gamma_{F D}$ is decided by $\gamma_{R}$ and $\gamma_{C}$ jointly. By getting the first class partial differential equation of $\gamma_{F D}$ in term of $\gamma_{R}$, namely, $\partial \gamma_{F D} / \partial \gamma_{R}$, we can get the expression as:

$$
\frac{\partial \gamma_{F D}}{\partial \gamma_{R}}=\frac{\gamma_{C}^{2}+1}{\left(\gamma_{C}+\gamma_{R}+1\right)^{2}}
$$

The equation above is obviously constant positive. Therefore, $\gamma_{F D}$ is monotonous increasing in terms of $\gamma_{R}$. We can derive that this is the case for $\gamma_{C}$ also. It is easy to understand from this result that the end-to-end SINR of two hops FD relay will be improved by either of any one link with better channel quality. So, for the sake of fulfilling the rate requirement of cellular, we should enhance one or both of $\gamma_{R}$ and $\gamma_{C}$.

Let us then analyze the corresponding received SINR in the D2D relay and in the destination of the cellular user which are expressed as shown in (12) and (15) respectively. In the first hop between BS and D2D relay, the SINR will be improved by decreasing the transmitting power of the relay due to the excessive loopback interference. In contrast, the SINR at the destination of the cellular user is a monotonous increasing function in terms of the transmitting power of relay. Then we know that there will be an optimal transmitting power of relay. In terms of power splitting factor of $\alpha$, the SINR at the destination of the cellular user will also be improved following $\alpha$ increasing. On the contrary, the SINR for D2D receiver will decrease as the spilt factor increases.

Now we provide a new insight for the objective function which considers fulfilling the rate requirement of the cellular user in the primary and improves the D2D transmitting rate in the secondary. We have observed that an optimal power at the D2D relay exists such that a higher or lower value will result in the deterioration of the end-to-end SINR of cellular user. Therefore, by getting the optimal transmitting power of relay, the energy consumption for D2D users who usually handle equipment such as a mobile phone will decrease. Moreover, our proposed scheme also makes sense of the demand of power split factor $\alpha$ at the D2D relay. Then the D2D relay can spare more power to transmit its own signals.

It should be made clear that the optimal transmitting power at the relay is not the global optimization as the achievable rate of D2D link is monotonously increased as follow $p_{R}$. We can demonstrate the first class partial differential of $\gamma_{D}$ in term of $p_{R}$ :

$$
\begin{aligned}
\frac{\partial \gamma_{D}}{\partial P_{R}} & =\frac{(1-\alpha) \gamma_{R D}\left(\gamma_{B D}+\alpha p_{R} \gamma_{R D}+1\right)-\alpha \gamma_{R D}(1-\alpha) p_{R} \gamma_{R D}}{\left(\gamma_{B D}+\alpha p_{R} \gamma_{R D}+1\right)^{2}} \\
& =\frac{(1-\alpha) \gamma_{R D}\left(\gamma_{B D}+1\right)}{\left(\gamma_{B D}+\alpha p_{R} \gamma_{R D}+1\right)^{2}}
\end{aligned}
$$


In the situation that $0 \leq \alpha \leq 1, \gamma_{R D}$ and $\gamma_{B D}$ is a constant greater than zero, so $\partial \gamma_{D} / \partial P_{R} \geq 0$ is permanently satisfied. It is obvious that the SINR will be greater as the transmitter power is larger when the channel to noise ratio is a constant.

Therefore, in order to improve the energy efficiency of D2D relay, we adapt the optimal power for guaranteeing cellular rate requirement which is equivalent to the minimum transmission power usage for D2D relay. The optimal FD relay transmission power is:

$$
p_{R}^{*}=\min \left\{1, \sqrt{\frac{\left(\gamma_{B R}+1\right)\left(\gamma_{B C}+1\right)}{\gamma_{L} \gamma_{R C}}}\right\}
$$

Proof: By substituting Equation (12), (15) into (16), the end-to-end SINR for cellular can be given by (we can convert into the following form):

$$
\gamma_{F D}=\frac{\gamma_{B R} \gamma_{R D} p_{R}}{\gamma_{R D} \gamma_{L} p_{R}^{2}+\gamma_{R D} p_{R}+\gamma_{B D} \gamma_{L} p_{R}+\gamma_{L} p_{R}+\gamma_{B R} \gamma_{B D}+\gamma_{B R}+\gamma_{B D}+1}
$$

by solving the one class partial differential equation of $\gamma_{F D}$ in terms of $p_{R}$, we can get the numerator expression of $\partial \gamma_{F D} / \partial p_{R}$ as:

$$
\gamma_{B R}^{2} \gamma_{R C} \gamma_{B C}+\gamma_{B R}^{2} \gamma_{R C}+\gamma_{B R} \gamma_{R C} \gamma_{B C}+\gamma_{B R} \gamma_{R C}-\gamma_{B R} \gamma_{L} \gamma_{R C}^{2} p_{R}^{2}=0
$$

which is equivalent to the one class partial differential equation $\partial \gamma_{F D} / \partial p_{R}$. The optimal transmission power can be obtained by solving the equation above.

Consequently, Equation (24) reveals that the maximum transmission power will be chosen, namely $p_{R}^{*}=1$ when:

$$
\gamma_{L} \leq \frac{\left(\gamma_{B R}+1\right)\left(\gamma_{B C}+1\right)}{\gamma_{R C}}
$$

the inequalities above show that the maximum transmit power is optimal only with weak loopback interference. Otherwise, the performance can be improved by backing off from the maximum power as reasoned before. In practice, we apply constrained power adaptation for which the SINRs coincide with those of the unconstrained case given the above whenever the conditions in (27) are not satisfied.

By further substituting $p_{R}^{*}$ into $\gamma_{F D}=\gamma$ where the equation guarantees the rate requirement of cellular we can obtain the optimal power split factor (also the minimum power the cellular share from the $\mathrm{D} 2 \mathrm{D}$ relay) as:

$$
\alpha^{*}=\min \left\{1, \frac{\gamma\left(\gamma_{B C}+1+\gamma_{R C} p_{R}^{*}\right)\left(\gamma_{B R}+1+\gamma_{L} p_{R}^{*}\right)}{2 \gamma_{B R} \gamma_{R C} p_{R}^{*}}\right\}
$$

Up to now, we have solved the optimal transmit powers allocated at the BS and at the D2D transmitter as well as the optimal power splitting factor at the D2D transmitter. The optimal power allocation could be found at the BS in a centralized manner, but the global channel state information is required. Via a dedicated feedback channel, e.g., the cognitive pilot channel proposed by the E2R2/E3 consortium in [12], the channel state information and the optimal power allocation can be conveyed between the BS and the distributed UE reliably.

\section{Simulation Results}

In this section, we show that the simulation results demonstrate high achievement transmit rate and power efficiency of the proposed FD relay-based D2D communication scheme and guarantee the rate requirement for the cellular user in the primary. We choose the HD relaying mode as a benchmark. It should be made clear that the spectrum band consumed in a traditional cellular system is double that of our underlaying D2D communication due to one orthogonal subchannel that is needed for the 
cellular user and D2D users respectively. Therefore, the D2D communication we proposed achieves an improvement of spectral efficiency for cellular system. In this section, we study relaying and transmit power adaptation in the case of static channels and, thus, exploiting instantaneous channel state information. Alternatively, the following analysis explains the performance during one instantaneous snapshot within channel coherence time in a slow-fading environment. The parameter of system as rate requirement for cellular is set as $R^{r e}=20 \mathrm{~kb} / \mathrm{s}$, which represents a typical voice signal characteristic and the bandwidth $W=0.1 \mathrm{MHz}$ which corresponds to the width of the subchannel allocated to one cellular user.

In Figure 2, we show the impact of loopback interference on the optimal transmission power of D2D relay by setting $\gamma_{B R}=\gamma_{R C}=10 \mathrm{~dB}$ and $\gamma_{B C}=\gamma_{B D}=-10 \mathrm{~dB}$ which means that channels from BS to cellular user and D2D receiver suffer from deep fading or shadowing. In the simulation, we change the channel gain of the loopback link at the D2D relay $\gamma_{L}$ from $5 \mathrm{~dB}$ to $30 \mathrm{~dB}$. It can be seen that the optimal transmit power is decreasing as the channel gain of loopback link is stronger. We can also observe that for a particular loopback gain, enlargement of the transmission power of relay is even harmful for the achievable rate for the cellular user which demonstrates our analysis we mentioned before. Therefore, in view of this problem, it is desirable to determine the optimal allocation power for the relay.

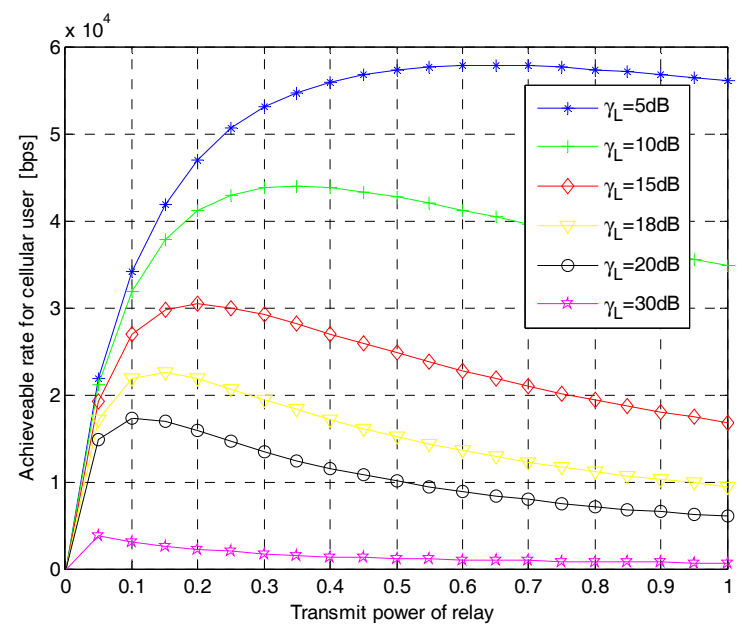

Figure 2. Achievable rate for cellular user with different transmission power usage.

In Figure 3, we observe the impact of power split factor on the system performance when we set $\gamma_{L}=10 \mathrm{~dB}$. As we can observe from Figure 3, in the range of $\alpha \geq 0.24$, the scheme we proposed could satisfy the rate requirement for the cellular while at the same time the achievable rate for D2D communication is appropriate. Furthermore, this scheme provides significant performance improvement in terms of the achievable sum rate of the system. It also can be seen that the choice of power split factor has a great significance on system performance. The transmit rate of the cellular user will increase with a large power split factor. However, enlarging power split factor will also lead to the deterioration of D2D communication performance. Therefore, the selection of power split factor for the relay node is important. 


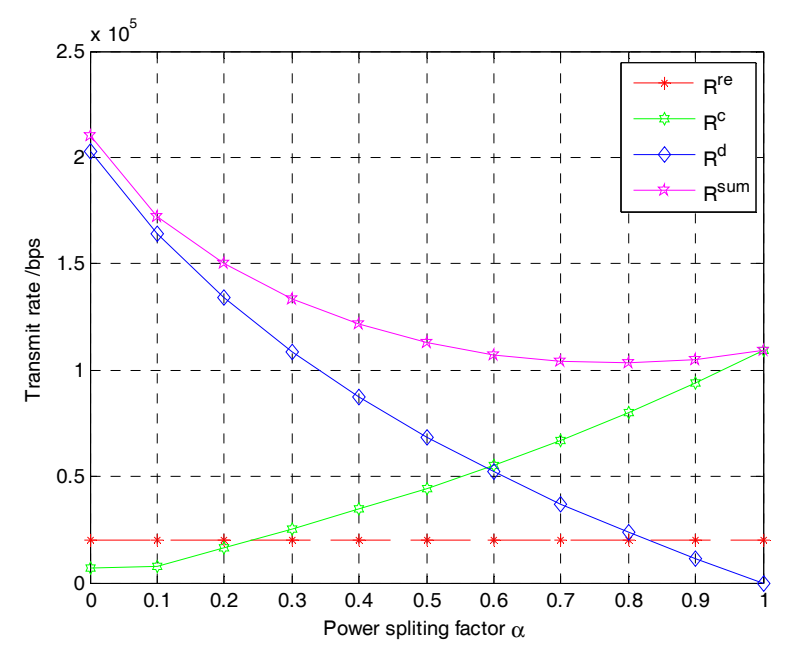

Figure 3. System performance with different power splitting factor.

In Figures 4 and 5, we make a comparison to the HD D2D relay mode in terms of optimal transmit power $p_{R}^{*}$ and power splitting factor $\alpha$. Due to transmitting signals in two orthogonal time slots, D2D relay in HD mode uses the maximum power as the optimal transmit power. In contrast, the scheme we proposed uses less power and guarantees the rate requirement for the cellular user which makes it much better in extending the battery life. It also can be observed that the optimal power is decreasing as the channel gain between D2D relay and cellular is stronger in our proposed scheme. Figure 5 shows that the power splitting factor gets smaller as the self-interference becomes weaker in FD mode, which means the D2D relay could share more of its power to support its own communication demand.

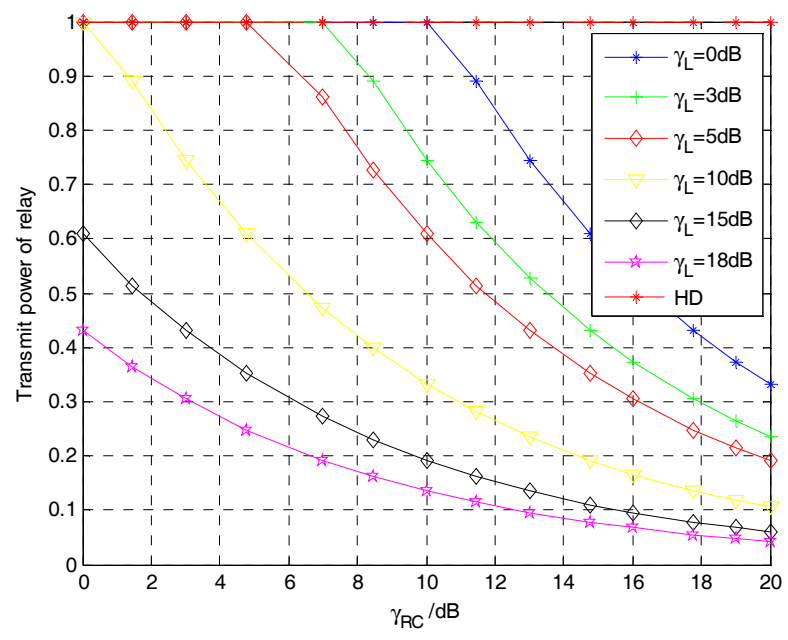

Figure 4. Optimal transmit power of relay.

In Figure 6, with the optimal transmit power and power split factor as we illustrated above, it is obvious that the D2D link can achieve significant rate gain in our proposed FD relaying-based D2D communication scheme. The simulation result also shows that the performance of a D2D achievable rate is significantly improved when $\gamma_{L}<15 \mathrm{~dB}$ and it also has a faster increase tendency than the benchmark of HD mode, even in the presence of $\gamma_{L}=15 \mathrm{~dB}$. The reason for this is that the FD relaying-based D2D scheme could transmit and receive signal simultaneously rather than occupy one of two orthogonal time slots for transmitting and receiving signal respectively. For this reason, the FD relaying based D2D communication scheme inherently has a much higher spectrum efficiency in comparison to HD mode. 


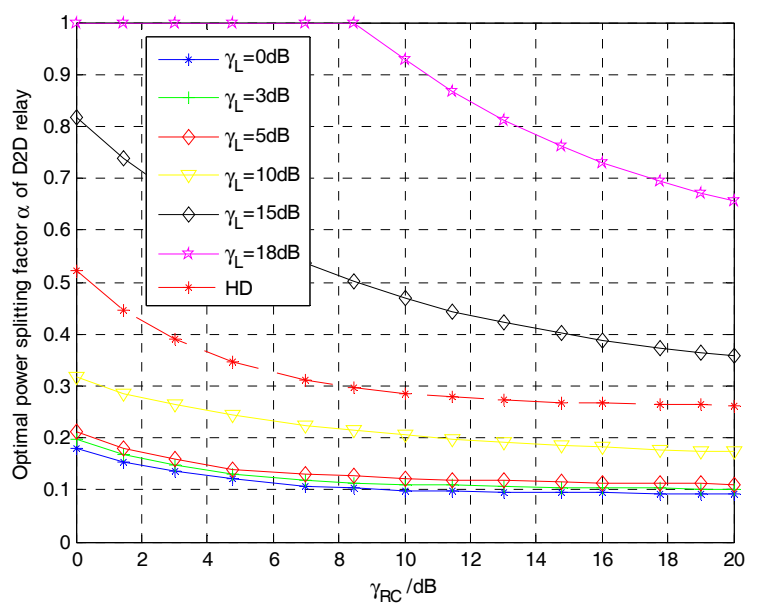

Figure 5. Optimal power splitting factor of relay.

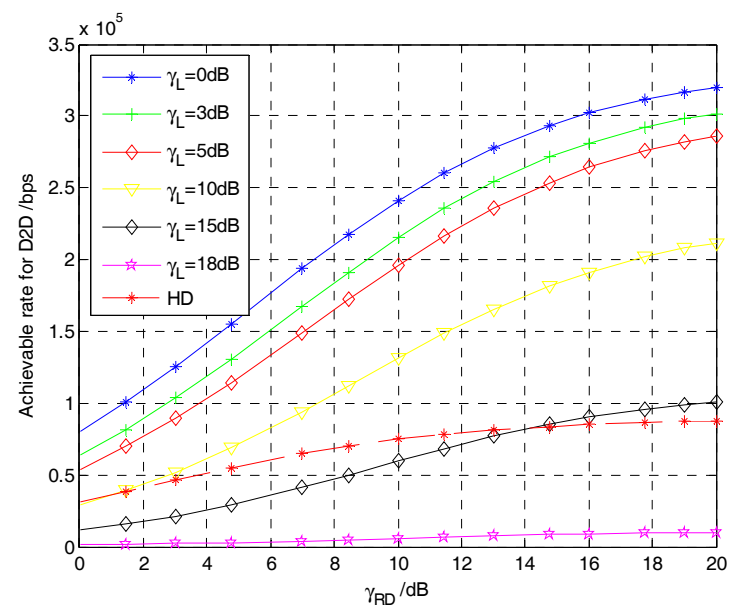

Figure 6. Achievable rate for D2D communication.

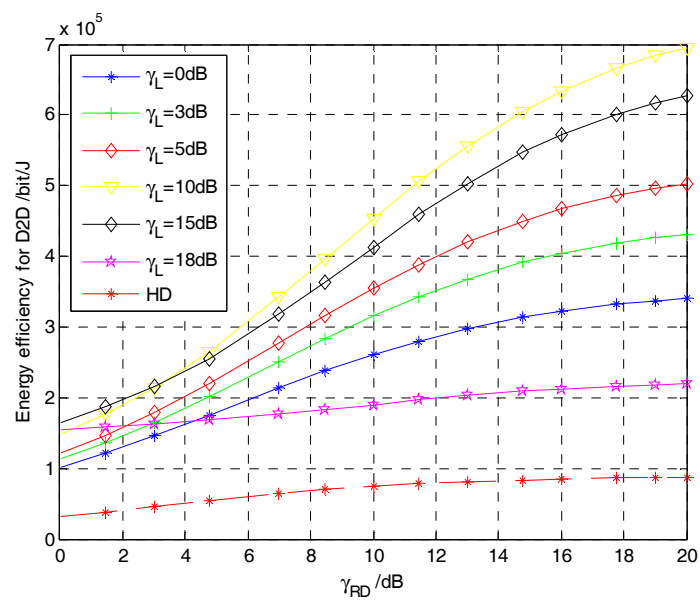

Figure 7. Energy efficient of D2D communication.

In contrast to Figure 6, Figure 7 reveals the energy efficiency of our proposed scheme when compared to the traditional HD mode. We can observe that the proposed scheme improves the energy efficiency of HD mode greatly. The reason for this is that the relay node of the HD mode forwards the cellular signal with maximum power usage for D2D relay, namely, $p_{R}^{*}=1$. However, the optimal 
power for the FD mode is always less than the maximum power and decreases as the channel gain for the loopback link increases. It also can be seen that the performance of energy efficiency does not improve with the mitigation of the self-interference of the relay. The reason for this result is that, upon efficient mitigation of self-interference of relay, the optimal power at D2D relay is increasing, and as we can see from Figure 4, this ultimately decreases the energy efficiency.

\section{Conclusions}

In this paper, we proposed a FD relaying based D2D communication scheme to assist the cellular communication by underlaying its downlink spectral resource. To fulfill the rate requirement of the cellular user by using the least power and to maximize the achievable rates for D2D link at the same time, we derive the optimal transmit power and power split factor for the D2D relay in a closed form. The simulation results show that the scheme we proposed brings significant achievable rate gain for D2D communication. Moreover, the scheme decreases transmit power usage by $80 \%$ for the D2D relay node in comparison to the HD mode, even in the presence of $\gamma_{L}=15 \mathrm{~dB}$ in Figure 4, which extends the lifetime of the battery and improves the energy efficiency of system, as shown in Figure 7.

Acknowledgments: This paper is funded by the International Exchange Program of Harbin Engineering University for Innovation-oriented Talents Cultivation. And this work is partially supported by National Natural Science Foundation of China (Grant No. 51509049), the Natural Science Foundation of Heilongiiang Province China (Grant No. F201345), the Fundamental Research Funds for the Central Universities of China (No. GK2080260140) and the National key research and development program (Grant No. 2016YFF0102806).

Author Contributions: Hui Dun Conceived, designed the experiments and performed the experiments; Fang Ye and Yibing Li analyzed the data; Fang Ye contributed reagents/materials/analysis tools; Hui Dun wrote the paper.

Conflicts of Interest: The authors declare no conflict of interest.

\section{References}

1. Lin, X.; Andrwes, J.; Ghosh, A.; Ratasuk, R. An overview of 3GPP device-to-device proximity services. IEEE Commun. Mag. 2014, 52, 40-48. [CrossRef]

2. Asadi, A.; Wang, Q.; Mancuso, V. A survey on device-to-device communication in cellular networks. IEEE Commun. Surv. Tutor. 2014, 16, 1801-1819. [CrossRef]

3. Doppler, K.; Rinne, M.; Wijting, C.; Ribeiro, C.; Hugl, K. Device-to device communication as an underlay to LTE-Advanced networks. IEEE Commun. Mag. 2009, 47, 42-49. [CrossRef]

4. Corson, M.; Laroia, R.; Li, J.; Park, V.; Richardson, T.; Tsirtsis, G. Toward proximity-aware internetworking. IEEE Wirel. Commun. Mag. 2010, 17, 26-33. [CrossRef]

5. Yang, M.J.; Lim, S.Y.; Park, H.J.; Park, N.H. Solving the data overload: Device-to-device bearer control architecture for cellular data offloading. IEEE Veh. Technol. Mag. 2013, 8, 31-39. [CrossRef]

6. Phunchongharn, P.; Hossain, E.; Kim, D. Resource allocation for device-to-device communications underlaying LTE-Advanced networks. IEEE Wirel. Commun. Mag. 2013, 20, 91-100. [CrossRef]

7. Feng, D.Q.; Lu, L.; Yuan-Wu, Y.; Li, G.; Li, S.; Feng, G. Device-to-device communications in cellular networks. IEEE Commun. Mag. 2014, 52, 49-55. [CrossRef]

8. Yu, C.H.; Doppler, K.; Ribeiro, C.; Tirkkonen, O. Resource sharing optimization for device-to-device communication underlaying cellular networks. IEEE Trans. Wirel. Commun. 2011, 10, 2752-2763.

9. Yu, G.D.; Xu, L.; Feng, D.; Yin, R.; Li, G.Y.; Jiang, Y. Joint mode selection and resource allocation for device-to-device communications. IEEE Trans. Commun. 2014, 62, 3814-3824. [CrossRef]

10. Ye, Q.; Al-Shalash, M.; Caramanis, C.; Andrews, J.G. Device-to-device modeling and analysis with a modified Matern hardcore BS location model. In Proceedings of the IEEE GLOBECOM, Atlanta, GA, USA, 9-13 December 2013; pp. 1825-1830.

11. Lin, X.; Andrews, J.G. Optimal spectrum partition and mode selection in device-to-device overlaid cellular networks. In Proceedings of the IEEE GLOBECOM, Atlanta, GA, USA, 9-13 December 2013; pp. 1837-1842.

12. Min, H.; Seo, W.; Lee, J.; Park, S.; Hong, D. Reliability improvement using receive mode selection in the device-to-device uplink period underlaying cellular networks. IEEE Trans. Wirel. Commun. 2011, 10, 413-418. [CrossRef] 
13. Jiang, Y.; Liu, Q.; Zheng, F.; Gao, X.; You, X. Energy-Efficient Joint Resource Allocation and Power Control for D2D Communications. IEEE Trans. Veh. Technol. 2016, 65, 6119-6127. [CrossRef]

14. Hakola, S.; Chen, T.; Lehtom, J.; Koskela, T. Device-to-device (D2D) communication in cellular network-performance analysis of optimum and practical communication mode selection. In Proceedings of the IEEE Wireless Communications and Networking Conference, Sydney, Australia, 18-21 April 2010.

15. Janis, P.; Koivunen, V.; Ribeiro, C.; Korhonen, J.; Doppler, K.; Hugl, K. Interference-aware resource allocation for device-to-device radio underlaying cellular networks. In Proceedings of the IEEE 69th Vehicular Technology Conference Spring, Barcelona, Spain, 26-29 April 2009.

16. Zhao, W.; Wang, S. Resource Allocation for Device-to-Device Communication Underlaying Cellular Networks: An Alternating Optimization Method. IEEE Commun. Lett. 2015, 19, 1398-1401. [CrossRef]

17. Xu, S.; Wang, H.; Chen, T.; Huang, Q.; Peng, T. Effective interference cancellation scheme for device-to-device communication underlaying cellular networks. In Proceedings of the IEEE Vehicular Technology Conference Fall, Ottawa, ON, Canada, 6-9 September 2010.

18. Chen, X.; Proulx, B.; Gong, X.; Zhang, J. Exploiting social ties for cooperative D2D communications: A mobile social networking case. IEEE/ACM Trans. Netw. 2015, 23, 1471-1484. [CrossRef]

19. Laneman, J.N.; Tse, D.N.C.; Wornell, G.W. Cooperative Diversity in Wireless Networks: Efficient Protocols and Outage Behavior. IEEE Trans. Inf. Theory 2004, 50, 3062-3080. [CrossRef]

20. Zheng, D.; He, C.; Jiang, L.; Ding, J.; Zhang, Q. Power Optimization for D2D Communication Based on Rate Requirement in Relay-Assisted Networks. In Proceedings of the 2015 IEEE International Conference on Communication Workshop (ICCW), London, UK, 8-12 June 2015; pp. 686-691.

21. Han, Y.; Pandharipande, A.; Ho Ting, S. Cooperative Spectrum Sharing via Controlled Amplify-and-Forward Relaying. In Proceedings of the IEEE Wireless Communications and Networking Conference (WCNC), Singapore, 11-13 December 2013; pp. 3346-3351.

22. Pei, Y.; Liang, Y.C. Resource allocation for device-to-device communications overlaying two-way cellular networks. IEEE Trans. Wirel. Commun. 2013, 12, 3611-3621. [CrossRef]

23. Zhou, B.; Hu, H.; Huang, S.Q.; Chen, H.H. Intracluster device-to-device relay algorithm with optimal resource utilization. IEEE Trans. Veh. Technol. 2013, 62, 2315-2326. [CrossRef]

24. Zhao, M.; Gu, X.; Wu, D.; Ren, L. A Two-Stages Relay Selection and Resource Allocation Joint Method for D2D Communication System. In Proceedings of the IEEE Wireless Communications and Networking Conference (WCNC), Doha, Qatar, 3-6 April 2016.

25. Han, Y.; Pandharipande, A.; Ho Ting, S. Cooperative Decode-and-Forward Relaying for Secondary Spectrum Access. IEEE Trans Wirel. Commun. 2009, 8, 4945-4950.

26. Zhang, G.; Yang, K.; Wu, S.; Mei, X.; Zhao, Z. Efficient power control for half-duplex relay based D2D networks under sum power constraints. Wirel. Netw. 2015, 21, 2345-2355. [CrossRef]

27. Zhang, G.; Yang, K.; Liu, P.; Wei, J. Power Allocation for Full-Duplex Relaying-Based D2D Communication Underlaying Cellular Networks. IEEE Trans. Veh. Technol. 2015, 64, 4911-4916. [CrossRef]

28. Riihonen, T.; Werner, S.; Wichman, R. Hybrid full-duplex/half-duplex relaying with transmit power adaptation. IEEE Trans. Wirel. Commun. 2011, 10, 3074-3085. [CrossRef]

29. Kim, T.; Dong, M. An Iterative Hungarian Method to Joint Relay Selection and Resource Allocation for D2D Communications. IEEE Wirel. Commun. Lett. 2014, 3, 625-628.

(c) 2017 by the authors. Licensee MDPI, Basel, Switzerland. This article is an open access article distributed under the terms and conditions of the Creative Commons Attribution (CC BY) license (http:/ / creativecommons.org/licenses/by/4.0/). 